\title{
Smoking Cessation Strategies Targeting Specific Populations
}

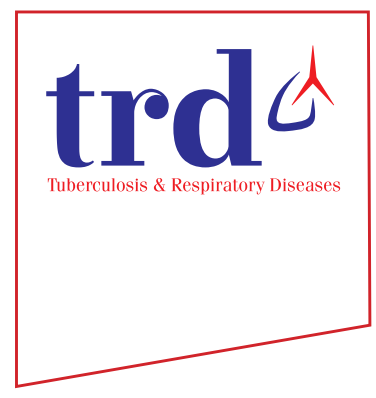

Eun Mi Chun, M.D., Ph.D. (10)

Division of Pulmonology and Critical Care Medicine, Department of Internal Medicine, Ewha Womans University Mokdong Hospital, Ewha Womans University College of Medicine, Seoul, Korea

Tuberc Respir Dis. 2019 Jan;82(1):1-5.

Published online Mar 7, 2018. https://doi.org/10.4046/trd.2017.0101

On page 3, "The control group of patients with no mental disorders showed no differences from other groups in the frequency of serious neuropsychiatric side effects, which were observed in 13 of the 99 subjects in the varenicline group (1.3\%), 22 of the 989 subjects in the bupropion group (2.2\%), 25 of the 1,006 subjects in the nicotine patch group (2.5\%), and 24 of the 999 subjects in the placebo group (2.4\%)." should be corrected as follows:

The control group of patients with no mental disorders showed no differences from other groups in the frequency of serious neuropsychiatric side effects, which were observed in 13 of the $\underline{\mathbf{9 9 0}}$ subjects in the varenicline group (1.3\%), 22 of the 989 subjects in the bupropion group (2.2\%), 25 of the 1,006 subjects in the nicotine patch group (2.5\%), and 24 of the 999 subjects in the placebo group (2.4\%). 